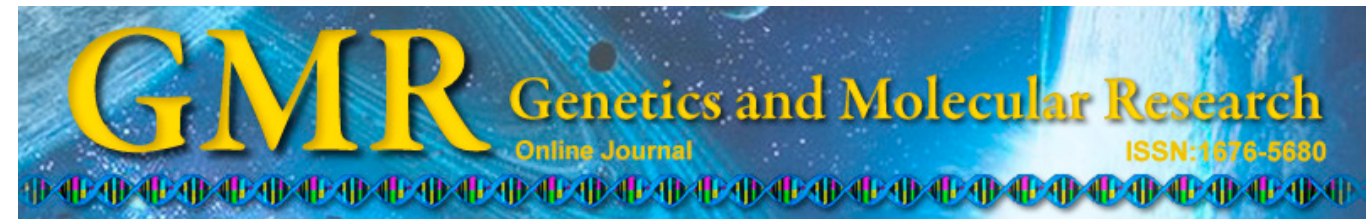

\title{
Differential microRNA expression in signet-ring cell carcinoma compared with tubular adenocarcinoma of human gastric cancer
}

\author{
F.Q. Li $^{1}$, B. Xu ${ }^{2}$, Y.J. Wu ${ }^{1}$, Z.L. Yang ${ }^{1}$ and J.J. Qian ${ }^{1}$ \\ ${ }^{1}$ Department of General Surgery, The First Affiliated Hospital, \\ Zhejiang University, Hangzhou, Zhejiang Province, China \\ ${ }^{2}$ Department of General Surgery, Renmin Hospital, \\ Hubei University of Medicine, Shiyan, Hubei Province, China \\ Corresponding author: Y.J. Wu \\ E-mail: ali901097@126.com
}

Genet. Mol. Res. 14 (1): 739-747 (2015)

Received January 3, 2014

Accepted March 20, 2014

Published January 30, 2015

DOI http://dx.doi.org/10.4238/2015.January.30.17

\begin{abstract}
Gastric cancer is a disease with a heterogeneous pathology; its pathological mechanisms remain unclear because there is a poor understanding of its etiology. In this study, we identified differentially expressed microRNAs (miRNAs) among various gastric cancer subtypes. miRNA microarray analysis and bioinformatic analysis were used to compare miRNA expression between the signet-ring cell carcinoma and tubular adenocarcinoma subtypes of gastric cancer. Thirteen dysregulated miRNAs were identified in signet-ring cell carcinoma compared with tubular adenocarcinoma: miR-30a, miR-26b, miR-381, let-7i, miR-29c, miR-543, miR-499-3p, miR-628-3p, miR524-5p, miR-181b, miR-1914, miR-663b, and miR-676. This is the first time that miR-499-3p, miR-628-3p, miR-524-5p, and miR-1914 have been identified in gastric cancer tissues. Bioinformatic analysis using target prediction algorithms indicated that these miRNAs are directly involved in gastric cancer pathogenesis and have different pathological mechanisms in various subtypes of signet-ring cell carcinoma and
\end{abstract}


tubular adenocarcinoma. The miRNA expression patterns in different gastric adenocarcinoma subtypes may help discriminate between signet-ring cell and tubular gland cancer or other gastric cancer subtypes that would otherwise be difficult to identify using routine histological and immunohistochemical analyses. These preliminary data should be verified in further prospective studies.

Key words: Gastric cancer; MicroRNA; Signet-ring cell carcinoma; Tubular adenocarcinoma

\section{INTRODUCTION}

On a microscopic level, the morphology of gastric cancer shows substantial variations in its histopathological differentiation. Among the various classification systems that have been developed, the most widely used definitions were proposed by the World Health Organization and by Lauren (1965) and Hamilton and Aaltonen (2000). In general, signet-ring cell carcinomas comprise the main fraction of the diffuse type of gastric cancer, as differentiated by Lauren (1965); by contrast, tubular carcinomas are the predominant fraction of the intestinal type. The former is characterized by liver metastasis and expansive growth, while the latter is characterized by peritoneal dissemination and infiltrative growth (Adachi et al., 2000; Arai et al., 2004). However, the pathological mechanisms leading to differences in their clinical and pathological features remain unclear.

Accumulating evidence indicates that aberrant microRNA (miRNA) expression contributes to carcinogenesis by promoting the expression of proto-oncogenes or by inhibition of the expression of tumor suppressor genes (Ambros, 2004; Bartel, 2004). Several studies have suggested that various miRNAs responsible for dysregulation of gastric cancer, such as miR-21, miR-27a, and miR-141, cause tumorigenesis and progression of gastric cancer (Du et al., 2009; Liu et al., 2009; Ueda et al., 2010). Whether the pathogenesis differs among the various subtypes of gastric cancer remains unclear. Using Lauren's (1965) classification of gastric cancer and miRNA microarray analysis, it has been reported that 8 miRNAs are up-regulated in the diffuse type of gastric cancer, whereas 4 miRNAs are up-regulated in the intestinal type of gastric cancer (Ueda et al., 2010). However, miRNA expression has not been reported using other classification systems, such as that proposed by the World Health Organization. miRNA profiling may clarify the differential pathological mechanisms of gastric cancer subtypes.

In the present study, we profiled the expression of miRNAs involved in tubular adenocarcinoma and compared these data with those obtained for signet-ring cell carcinoma. To determine whether the miRNA expression signatures differed, the validation of the microarray results was carried out for the most highly dysregulated miRNAs using quantitative reverse transcription-polymerase chain reaction (qRT-PCR). Moreover, we analyzed their potential differential contribution to the pathogenesis of gastric cancer histological subtypes followed by bioinformatic analysis. Our findings will increase the understanding of gastric carcinogenesis and be useful for identifying novel molecular markers that can be used to improve the individualized treatment of gastric cancer. 


\section{MATERIAL AND METHODS}

\section{Patients and specimen collection}

All human tissue samples were obtained from surgical specimens of 6 patients with gastric cancer, who had not undergone chemotherapy or radio-chemotherapy before gastrectomy, in 2011, at the First Affiliated Hospital of Zhejiang University College of Medicine, China. These fresh tissues, dissected from both gastric cancer and adjacent normal non-tumor tissues from the same patient, were collected in cryotubes and stored at $-70^{\circ} \mathrm{C}$ for future use. Nontumor tissues were $1.5 \mathrm{~cm}$ from the tumor; no obvious tumor cells were present. All diagnoses were verified by a pathologist. As per the AJCC International Tumor Node Metastasis Staging System (2010), all tumors were considered to be stages II-III. Histological classification of gastric cancer was established according to World Health Organization criteria (Hamilton and Aaltonen, 2000). Information regarding the gastric cancer specimens is presented in Table 1. The present study was approved by the Human Research Ethics Committee of Zhejiang University College of Medicine, and all the patients provided informed consent.

\begin{tabular}{|c|c|c|c|c|c|c|c|}
\hline \multirow[t]{2}{*}{ Samples } & \multirow[t]{2}{*}{ Gender } & \multirow[t]{2}{*}{ Age (years) } & \multirow[t]{2}{*}{ Location of tumor } & \multicolumn{2}{|c|}{ Histological types } & \multirow[t]{2}{*}{ Grade } & \multirow{2}{*}{$\begin{array}{c}\text { Cancer } \\
\text { stage (TMN) }\end{array}$} \\
\hline & & & & Lauren (1965) & WHO & & \\
\hline GC-1-1 & $\mathrm{F}$ & 86 & Antrum & Intestinal & Tubular & Moderate differentiation & II c \\
\hline $\mathrm{GC}-1-2$ & M & 59 & Body & Intestinal & Tubular & Moderate differentiation & IIIIa \\
\hline GC-1-3 & $\mathrm{F}$ & 72 & Angle & Intestinal & Tubular & Low-moderate differentiation & II c \\
\hline GC-2-1 & M & 68 & Body & Diffuse & Signet-ring cell & Low-moderate differentiation & III a \\
\hline GC-2-2 & $\mathrm{F}$ & 66 & Body & Diffuse & Signet-ring cell & Low differentiation & III b \\
\hline GC-2-3 & M & 42 & Antrum & Diffuse & Signet-ring cell & Low differentiation & IIIb \\
\hline
\end{tabular}

F, female; M, male; GC-1, gastric cancer set 1; GC-2, gastric cancer set 2.

\section{miR-enriched and total RNA extraction procedures}

Total RNA was extracted from the frozen tissues of 6 gastric cancer and normal gastric mucosa specimens using TRIzol reagent (Invitrogen, Carlsbad, CA, USA) according to manufacturer instructions, and miR-enriched RNA for low-density array analysis was isolated using the mirNeasy mini Kit (QIAGEN, Hilden, Germany). The quality and quantity of extracted RNA were measured using a NanoDrop spectrophotometer (ND-1000; NanoDrop Technologies, Wilmington, DE, USA) and RNA integrity was assessed by electropherogram and gel analysis.

\section{miRNA microarray assay}

After RNA isolation, miRNA was labeled using the miRCURY'TM $\mathrm{Hy} 3^{\mathrm{TM}} / \mathrm{Hy} 5^{\mathrm{TM}}$ Power labeling kit (Exiqon, Vedbaek, Denmark) according to manufacturer instructions. Next, the Hy $3^{\mathrm{TM}}$-labeled samples were hybridized onto miRCURY ${ }^{\mathrm{TM}}$ LNA Array slides (v.14.0) (Exiqon) following the array manual instructions. Following hybridization, the slides were processed and scanned using an Axon GenePix 4000B microarray scanner (Axon Instruments, Foster City, CA, USA). Scanned images were then imported into the GenePix 
Pro v. 6.0 software (Axon Instruments) for grid alignment and data extraction. Replicated miRNAs were averaged and miRNAs with intensities $>50$ were used for the calculation of the normalization factor. Expressed data were then normalized using median normalization. To identify significantly differently expressed miRNAs, we performed Volcano Plot filtering between the 2 groups. As thresholds to screen for up- or down-regulated miRNAs, those with fold-change $\geq 2.0$ and $\mathrm{P}$ value $\leq 0.05$ were determined. Hierarchical clustering was performed using the MEV software v. 4.6 (Institute for Genomic Research).

\section{qRT-PCR}

Quantitative miRNA RT-PCR was performed using the TaqMan microRNA Assay ${ }^{\mathbb{B}}$ kit according to manufacturer instructions (Applied Biosystems). The miRNA transcripts of 4 of the most highly dysregulated miRNAs (Hsa-miR-181b, Hsa-let-7i, Hsa-miR-663b, and HsamiR-676) were reverse-transcribed into cDNA using gene-specific miRNA qRT-PCR primer sets. Two sets of gastric signet-ring cell carcinoma and tubular adenocarcinoma samples were subjected to real-time PCR amplification for 40 cycles using an ABI7500 Standard system and miRNA-specific probes (Applied Biosystems), and were used for qRT-PCR analysis. Expression values were normalized to those of a small nucleolar RNA, RNU44 (Applied Biosystems). Foldchange is reported as a $\log _{2}$ value consistent with the miRNA microarray output format.

\section{Bioinformatic analysis}

A total of 4 software tools were employed to perform bioinformatic-based target prediction analysis: miRBAse Targets V4 (http://microrna.sanger.ac.uk/targets/v4/), PicTar predictions (http://pictar.bio.nyu.edu/), miRanda (http: //www. microrna.org/), and TargetScan 6.2 (http://www.targetscan.org/). A prediction was considered to be positive only if it was detected by at least 2 of the programs. miRNAs with the same mature sequences were treated as 1 miRNA. The chromosomal localization of aberrant regulation of miRNAs was assigned using the miRBAse database (miRBAse 18, http://microrna.sanger.ac.uk/sequences/). Some miRNAs, including miR-499-3p, miR-543, miR-628-3p, and miR-524-5p, were excluded from the present analysis because they have not been identified in other studies.

\section{RESULTS}

\section{miRNA microarray}

Using miRNA microarrays, 13 miRNAs that were differentially expressed between signet-ring cell carcinoma and tubular adenocarcinoma tissues were identified. The Volcano Plot filtering application was used to identify the 10 most important miRNAs that were up-regulated in signet-ring cell carcinomas and 3 miRNAs that were down-regulated compared with those in tubular adenocarcinoma. Table 2 shows differentially expressed miRNAs with at least 2 -fold-change in expression as suggested by the significance analysis of microarrays. Novel miRNA genes in tumor tissue include miR-499-3p, miR-499-3p, miR-524-5p, and miR-1914. Subsequently, hierarchy clustering was performed based on the normalized miRNA data, and those with foreground-background intensities $<50$ in all samples were excluded. Figure 1 shows the results of hierarchical clustering of miRNA expression profiles. 
Table 2. miRNAs differentially expressed in signet-ring cell carcinomas compared with tubularadenocarcinoma".

\begin{tabular}{lccc}
\hline has-miRNA & Fold-change (signet-ring cell vs tubular) & Differential expression & P value \\
\hline has-miR-30a & 3.739647 & $\mathrm{Up}$ & 0.015339 \\
has-miR-26b & 3.227654 & $\mathrm{Up}$ & 0.03609 \\
has-miR-381 & 3.765302 & $\mathrm{Up}$ & 0.018524 \\
has-let-7i & 2.445987 & $\mathrm{Up}$ & 0.035362 \\
has-miR-29c & 4.290275 & $\mathrm{Up}$ & 0.016269 \\
has-miR-543 & 3.182887 & $\mathrm{Up}$ & 0.000392 \\
has-miR-499-3p & 2.275241 & $\mathrm{Up}$ & 0.020798 \\
has-miR-628-3p & 2.143933 & $\mathrm{Up}$ & 0.045112 \\
has-miR-524-5p & 2.278742 & $\mathrm{Up}$ & 0.04342 \\
has-miR-181b & 2.427085 & $\mathrm{Up}$ & 0.022303 \\
has-miR-1914 & 0.496235 & Down & 0.043822 \\
has-miR-663b & 0.39109 & Down & 0.03667 \\
has-miR-676 & 0.200097 & Down & 0.003237 \\
\hline
\end{tabular}

\#Only microRNAs with high signals are shown here.

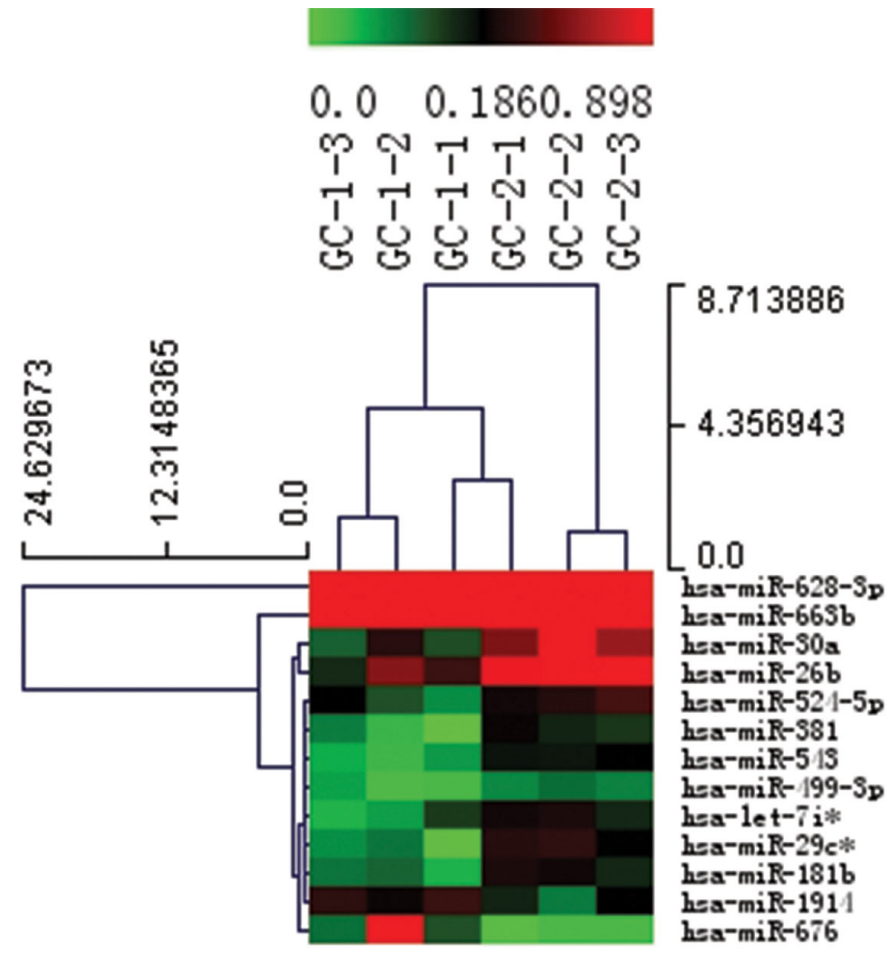

Figure 1. Hierarchical clustering of normalized miRNA data. Red indicates high relative expression, while green indicates low expression.

\section{Experimental validation}

To confirm the microarray findings, the expression levels of the 4 most dysregulated miRNAs, miR-181b, let-7i, miR-676, and miR-663b, in the 2 groups of signet-ring cell carcinomas were evaluated for tubular adenocarcinomas by qRT-PCR. The $\log _{2}$ fold-change values for the 4 miRNAs (Figure 2) were consistent with the microarray data (Table 2). 


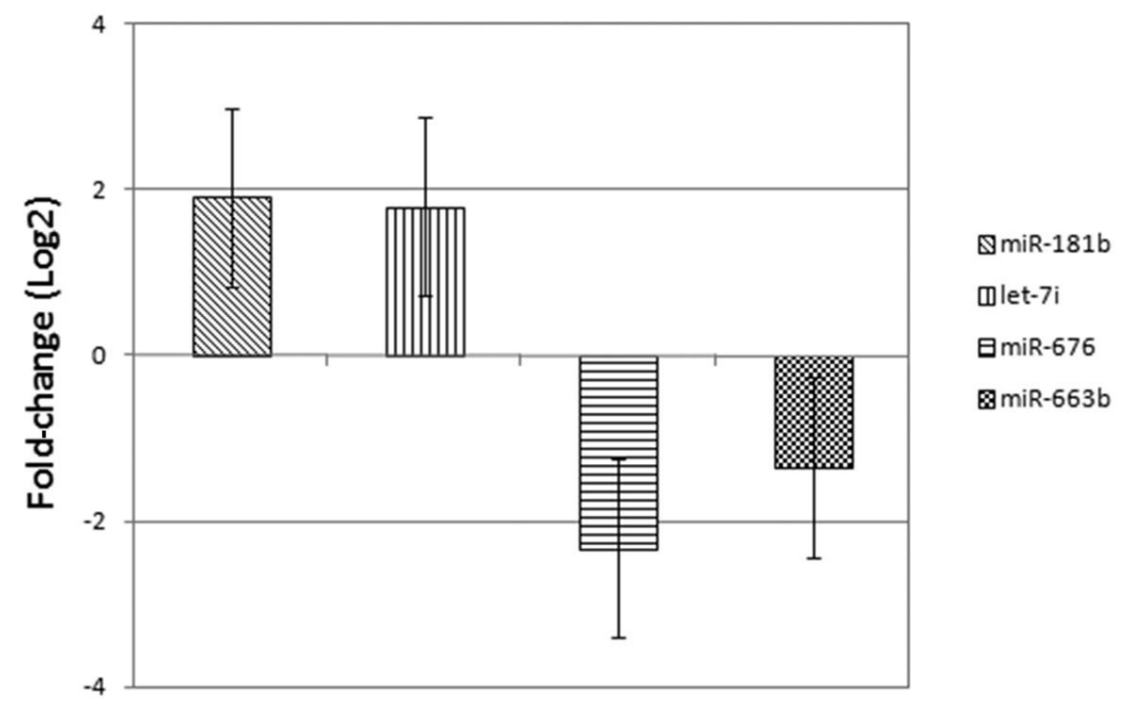

Figure 2. qRT-PCR validation of the expression of miR-181b, let-7i, miR-676, and miR-663b in signet-ring cell carcinomas $v s$ tubular adenocarcinomas. A positive value denotes an increase in cancer while a negative value denotes a decrease.

\section{Target prediction analysis}

To understand the involvement of miRNAs in the pathogenesis of different histological subtypes of gastric cancer, we identified potential targets of the 4 dysregulated miRNAs (Table 3) using 4 software tools. As shown previously, let-7i, whose chromosomal locus is chromosome 12q14.1, could be used to identify the more vulnerable vascular invasion and liver metastasis nature of signet-ring cell carcinomas compared to tubular adenocarcinomas because of CCND2 (G1/S-specific cyclin-D2), which is one of its predicted target genes. It was observed that the dysregulation of let-7i may be involved in this induction. These results showed that at least some miRNA differential expression could be attributed to the differential clinical and pathological behavior. However, this is not the only mechanism of regulation of miRNA expression in cancer.

Table 3. Expression profiles of miRNA in signet-ring cell carcinoma $v s$ tubular adenocarcinoma tissues and the chromosomal loci and predicted targets of these miRNAs.

\begin{tabular}{|c|c|c|}
\hline miRNA & Chromosomal locus & Predicted target genes \\
\hline $\begin{array}{l}\text { Up-regulated } \\
\text { miR-181b }\end{array}$ & $1 \mathrm{q} 32.1,9 \mathrm{q} 33.3$ & $\begin{array}{l}\text { TCLIA, VSNL1, GRIA2, KAT2B (PCAF), AICDA (AID), CDX2, GATA6, } \\
\text { NLK, NFAT5, ARHGEF3, CPOX, ACVR2B, RAPIB, TCERG1, TBLIX, } \\
\text { ATM, ACVR } 2 A \text {, and TNF. }\end{array}$ \\
\hline let-7i & $12 \mathrm{q} 14.1$ & $\begin{array}{l}\text { BTBD3, SOX13, SLC25A4, SEMA4F, RPUSD2, PLEKHG6, CCND2, } \\
\text { and TRIM41. }\end{array}$ \\
\hline $\begin{array}{l}\text { Down-regulated } \\
\text { miR-676 }\end{array}$ & $\mathrm{Xq13.1}$ & 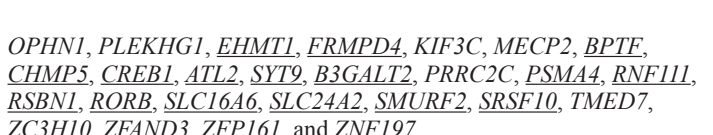 \\
\hline $\operatorname{miR}-663 b$ & $2 q 21.2$ & ZNF436, HMGN3, MAP2K7, and TNFSF 11. \\
\hline
\end{tabular}




\section{DISCUSSION}

This is the first report to compare signet-ring cell carcinomas and tubular adenocarcinomas using an miRNA microarray assay and to identify miRNAs whose expression was significantly down- or up-regulated using qRT-PCR validation. The results of this study will increase understanding of the involvement of miRNAs in different gastric cancer subtypes and reveal new biomarkers and targeted therapy options. For miRNAs identified by microarray analysis (Table 2 ), $\sim 77 \%$ were significantly up-regulated ( $>2$-fold), while the remaining miRNAs were down-regulated $(<1 / 2)$ in signet-ring cell carcinoma compared with tubular adenocarcinoma tissues. miR-181b, miR-29c, miR-381, and miR-26b showed the highest up-regulation, whereas miR-663b and miR-676 were the most downregulated. Among the miRNAs significantly differentially expressed, some are reportedly associated with other diseases, while miR-524-5p, miR-628-3p, miR-499-3p, and miR-1914 are not. Compared to other studies of gastric cancer, such as those comparing intestinal and diffuse types, differentiated and undifferentiated types, and gastric cancer and normal tissue, the miRNA differential expression profile of signet-ring cell carcinomas $v s$ tubular adenocarcinomas differed. These data suggest that the pathology of gastric cancer is complex, and the subtypes analyzed using the various classification methods differed significantly; thus, additional studies are needed to refine our results.

The miR-181 family participates in cell cycle progression and cell survival. In addition, patients with higher levels of miR-181 were more likely to show lymph node metastasis, vascular invasion by tumor cells, and poor prognosis (Yang et al., 2011). As a member of the miR-181 family, miR-181b also may play a role in the variation in tumorigenesis among different cancers, and its decreased expression is inversely correlated with increased protein levels of the myeloid cell leukemia sequence 1- and B-cell lymphoma 2-targeted genes ( $\mathrm{Ji}$ et al., 2009; Chen et al., 2010). miR-181b may also contribute to drug resistance of tamoxifen in breast cancer and tumor progression of gastric carcinomas (Jiang et al., 2011; Lu et al., 2011; Visone et al., 2012). In our study, the higher expression of miR-181b in signet-ring cell carcinoma compared with that in tubular adenocarcinoma suggests a correlation with the more malignant clinical behavior of signet-ring cell carcinoma. Thus, future studies should assess the mechanisms underlying the effects of miR-181b in gastric cancer subtypes. In the present study, one of the most highly expressed miRNAs in signet-ring cell carcinoma was hsa-let-7i, which was further validated by qRT-PCR in gastric cancer tissues. Hsa-let-7i is a new member of the let-7 miRNA family. Although little is known regarding its function, recent data have suggested that let-7i is a novel biomarker and therapeutic target in human epithelial ovarian cancer and colorectal cancer (Lui et al., 2007; Zhang et al., 2012). In the present study, the targets of hsa-let-7i were predicted using 2 programs, TargetScan and PicTar. Eight genes, TRIM41, SOX13, SLC25A4, SEMA4F, RPUSD2, PLEKHG6, CCND2, and BTBD3, were identified as hsa-let-7i targets. It has been reported that $C C N D 2$ (G1/S-specific cyclin-D2) is correlated with cancer invasion, lymph node metastasis, liver metastasis, and poor prognosis (Mermelshtein et al., 2005; Sarkar et al., 2010). Our data suggest that hsa-let-7i has a substantial clinical impact on different subtypes of gastric cancer. Further studies are necessary to shed mechanistic insight into the function of hsa-let-7i, as well as to establish its possible clinical application. In particular, continued investigation into the role of hsa-let-7i in gastric cancer is likely to yield important information on novel targets that can be used for individualized therapy, diagnosis, and prognosis. Thus, our findings may assist in the identification of 
miRNAs as novel biomarkers in gastric cancer metastasis and the translation of experimental findings into clinical applications.

Some of the miRNAs identified to be down-regulated in signet-ring cell carcinomas compared with tubular carcinomas are consistent with other reports, such as hsa-miR-676 and hsa-miR-663b. Interestingly, miR-676 has been identified as a target of p53 and is directly or indirectly regulated by the level of functional p53 in human neuroblastoma and mouse models (Terrile et al., 2011). These results indicate that down-regulation of this miRNA contributes to a more aggressive phenotype of the neuroblastoma tumor phenotype. In the present study, lower expression of miR-676 in signet-ring cell carcinomas correlates with more malignant clinical behavior. This is an interesting possibility requiring further studies. In addition, the level of miR-663b was also found to be significantly lower in signet-ring cell carcinoma. However, some studies reported that miR-663b may also be up-regulated in cutaneous T-cell lymphoma (Ralfkiaer et al., 2011). These results indicate that the functions of miRNAs are complex.

\section{CONCLUSIONS}

The miRNA expression patterns differed in various gastric adenocarcinoma subtypes; these results contribute to our understanding of the role of miRNAs in the pathogenesis of human gastric cancer. Such patterns may help to discriminate between signet-ring cell and tubular gland cancer or other gastric cancer subtypes that would otherwise be difficult to distinguish B-cell lymphoma 2 using routine histological and immunohistochemical analyses. More importantly, our data may facilitate personalized treatment of patients with dissimilar gastric cancer subtypes. However, these preliminary data should be verified in further prospective studies.

\section{Conflicts of interest}

The authors declare no conflict of interest.

\section{ACKNOWLEDGMENTS}

Research supported by the National Natural Science Funds of China (\#81372624), and the Health Department of Zhejiang Province (\#2012KYA070).

\section{REFERENCES}

Adachi Y, Yasuda K, Inomata M, Sato K, et al. (2000). Pathology and prognosis of gastric carcinoma: well versus poorly differentiated type. Cancer 89: 1418-1424.

AJCC Cancer Staging Manual (2010). International Tumor Node Metastasis Staging System. Edge S, Byrd DR, Compton CC, Fritz AG, et al., eds. Springer, New York.

Ambros V (2004). The functions of animal microRNAs. Nature 431: 350-355.

Arai T, Esaki Y, Inoshita N, Sawabe M, et al. (2004). Pathologic characteristics of gastric cancer in the elderly: a retrospective study of 994 surgical patients. Gastric Cancer 7: 154-159.

Bartel DP (2004). MicroRNAs: genomics, biogenesis, mechanism, and function. Cell 116: 281-297.

Chen G, Zhu W, Shi D, Lv L, et al. (2010). MicroRNA-181a sensitizes human malignant glioma U87MG cells to radiation by targeting Bcl-2. Oncol. Rep. 23: 997-1003.

Du Y, Xu Y, Ding L, Yao H, et al. (2009). Down-regulation of miR-141 in gastric cancer and its involvement in cell growth. J. Gastroenterol. 44: 556-561. 
Hamilton SR and Aaltonen LA (2000). World Health Organization Classification of Tumours. Pathology and Genetics of Tumours of the Digestive System. IARC Press, Lyon.

Ji J, Yamashita T, Budhu A, Forgues M, et al. (2009). Identification of microRNA-181 by genome-wide screening as a critical player in EpCAM-positive hepatic cancer stem cells. Hepatology 50: 472-480.

Jiang J, Zheng X, Xu X, Zhou Q, et al. (2011). Prognostic significance of miR-181b and miR-21 in gastric cancer patients treated with S-1/Oxaliplatin or Doxifluridine/Oxaliplatin. PLoS One 6: e23271.

Lauren P (1965). The two histological main types of gastric carcinoma: diffuse and so-called intestinal-type carcinoma. An attempt at a histo-clinical classification. Acta Pathol. Microbiol. Scand. 64: 31-49.

Liu T, Tang H, Lang Y, Liu M, et al. (2009). MicroRNA-27a functions as an oncogene in gastric adenocarcinoma by targeting prohibitin. Cancer Lett. 273: 233-242.

Lu Y, Roy S, Nuovo G, Ramaswamy B, et al. (2011). Anti-microRNA-222 (anti-miR-222) and -181B suppress growth of tamoxifen-resistant xenografts in mouse by targeting TIMP3 protein and modulating mitogenic signal. J. Biol. Chem. 286: 42292-42302.

Lui WO, Pourmand N, Patterson BK and Fire A (2007). Patterns of known and novel small RNAs in human cervical cancer. Cancer Res. 67: 6031-6043.

Mermelshtein A, Gerson A, Walfisch S, Delgado B, et al. (2005). Expression of D-type cyclins in colon cancer and in cell lines from colon carcinomas. Br. J. Cancer 93: 338-345.

Ralfkiaer U, Hagedorn PH, Bangsgaard N, Lovendorf MB, et al. (2011). Diagnostic microRNA profiling in cutaneous T-cell lymphoma (CTCL). Blood 118: 5891-5900.

Sarkar R, Hunter IA, Rajaganeshan R, Perry SL, et al. (2010). Expression of cyclin D2 is an independent predictor of the development of hepatic metastasis in colorectal cancer. Colorectal Dis. 12: 316-323.

Terrile M, Bryan K, Vaughan L, Hallsworth A, et al. (2011). miRNA expression profiling of the murine TH-MYCN neuroblastoma model reveals similarities with human tumors and identifies novel candidate miRNAs. PLoS One 6: e28356.

Ueda T, Volinia S, Okumura H, Shimizu M, et al. (2010). Relation between microRNA expression and progression and prognosis of gastric cancer: a microRNA expression analysis. Lancet Oncol. 11: 136-146.

Visone R, Veronese A, Balatti V and Croce CM (2012). MiR-181b: new perspective to evaluate disease progression in chronic lymphocytic leukemia. Oncotarget 3: 195-202.

Yang CC, Hung PS, Wang PW, Liu CJ, et al. (2011). miR-181 as a putative biomarker for lymph-node metastasis of oral squamous cell carcinoma. J. Oral Pathol. Med. 40: 397-404.

Zhang P, Ma Y, Wang F, Yang J, et al. (2012). Comprehensive gene and microRNA expression profiling reveals the crucial role of hsa-let-7i and its target genes in colorectal cancer metastasis. Mol. Biol. Rep. 39: 1471-1478. 